this study were to analyse the clinical impact of morbidities and to identify predictors of in-hospital mortality in preterm infants with low birth weight.

Methods Between January-2011 and November-2012 were included 81 preterm infants at our centre with low birth weight or $\leq 32$ weeks gestation. Perinatal variables were included in risk factor analysis. Data are expressed as gestational age (GA) $<28$ or between $28-32$ weeks and birth weight defined as: low $<2500$ grams, very low 1000-1500 grams and extremely $<1000$ g. Results: The mean GA was $29 \pm 2$ (23-32 weeks) and mean birth weight was $1230.8 \pm 368(510-2000 \mathrm{~g})$. The neonatal mortality rate was $17.3 \%$. Preterm infants who died had lower birth weight than were alive, $797 \pm 249$ vs. $1332 \pm 315, \mathrm{p}<$ 0,001 . The overall incidence of respiratory distress syndrome was $86.3 \%$, septicaemia $24.7 \%$, neurological damage $18.5 \%$ and necrotizing enterocolitis was 7.4\%. The SNAP II, SNAPPE II and CRIB II scores showed a high discriminatory power for predicting hospital mortality, ROC area $0.863,0.925$ and 0.925 , $\mathrm{p}<0.001$, respectively. Multivariate analysis of predictors of inhospital mortality were necrotizing enterocolitis, risk scores, low 5-min Apgar score, inotropic support, and protectors were: the absence of intraventricular haemorrhage, cardiopulmonary resuscitation and increased GA.

Conclusion The survival in preterm infants in addition of GA or birth weight, it depends on the presence of morbidities. The use of risk scores on admission is useful for prediction in-hospital mortality.

\section{PO-0640 CLINICAL IMPACT OF MATERNAL CHARACTERISTICS IN PRETERM INFANTS WITH LOW BIRTH WEIGHT}

M Muñoz-Garcia, G Viedma-Chamorro, C Santiago-Gutiérrez, MC Martínez-Padilla, J de la Cruz-Moreno. Department of Pediatrics, Complejo Hospitalario de Jaén, Jaén, Spain

\subsection{6/archdischild-2014-307384.1281}

Maternal risk factors can cause prematurity. The aim of our study was to analyse the impact of maternal characteristics in preterm low birth weight.

Methods A retrospective cohort study of a total of 81 preterm infants at our centre with low birth weight and $\leq 32$ weeks of gestation was performed. We analysed perinatal risk factors. Data are expressed as a function of gestational age (GA) $<28$ or between 28-32 weeks and birth weight defined as: extremely $<1000 \mathrm{~g}$, very low 1001-1500 g and low weight 1501-2000 g.

Results The mean maternal age was $30.3 \pm 5.8$ years. There were no statistically significant differences when comparing maternal age in according to birth weight $(29.2 \pm 5.7$ vs. 31.5 \pm 6.6 vs. $30.2 \pm 4.6, p=0.460$ respectively) or gestational age (30.4 \pm 5 vs. $30.3 \pm 6, p=0.964)$. The presence of maternal risk factors was very low: $3.7 \%$ were smokers, $2.5 \%$ had obesity. There were preeclampsia and diabetes gestational in 5 and 3 cases, respectively. Chorioamnionitis was diagnosed in $13.6 \%$ of pregnancies and $71.3 \%$ received antenatal steroids. The pregnancy was twins in $29.6 \%$ of cases. No significant differences were found when perinatal characteristics were examined in terms of birth weight, except for gestational diabetes (0\% vs. $0 \%$ vs. $13.6 \%$ in low birth, $\mathrm{p}=0,016)$. Maternal age did not influence in neonatal mortality $(30.2 \pm 5$ years in preterm infants living vs. $30.7 \pm 6$ years in those died, $\mathrm{p}=0.784$.

Conclusions In our series, the presence of maternal risk factors was very low. We were not found significant differences in according to birth weight or impact on neonatal mortality.

\section{PO-0641 PAEDIATRIC SUBGLOTTIC CYSTS : RISK FACTORS AND PREVENTION - CASE CONTROL STUDY}

${ }^{1} \mathrm{CH} \mathrm{Ng},{ }^{2} \mathrm{JM}$ Wood, ${ }^{3} \mathrm{~S}$ Rao, ${ }^{2} \mathrm{~S}$ Vijayasekaran. ${ }^{1}$ Paediatric Medicine, Princess Margaret Hospital for Children, Perth, Australia; ${ }^{2}$ Otorhinolaryngology, Princess Margaret Hospital for Children, Perth, Australia; ${ }^{3}$ Neonatal and Perinatal Medicine, Princess Margaret Hospital for Children, Perth, Australia

\subsection{6/archdischild-2014-307384.1282}

Background Subglottic cysts (SGCs) are a rare, but important cause of stridor in children. They can cause significant upper airway obstruction, with potentially lethal consequences, particularly when exacerbated by upper respiratory tract infection. Although seen typically in pre-term infants, there is a paucity of literature regarding their aetiology.

Aim To examine the background characteristics and to determine any risk factors in paediatric patients with SGCs.

Methods A retrospective case control study was conducted, reviewing reports of all laryngotracheobronchoscopies (LTB) conducted in a tertiary paediatric hospital, between January 2009 and 2014. SGCs were identified in 15 patients from the 1095 LTBs performed. Cases were matched to controls based on gestational age at birth, birth weight and age. Medical records of cases and controls were reviewed to identify possible risk factors.

Results Of the 15 patients with SGCs, 14 were born prematurely. All of the patients with SGCs had been ventilated, and 13 of the 15 controls were ventilated. Overall time of ventilation and frequency of re-intubations were similar between the two groups, however SGCs patients had significantly higher frequency of ETT suctioning conducted during period of ventilation.

Conclusion This study demonstrated an association of SGCs in the pre-term infants with previous intubation. SGCs were more prevalent in infants that required more frequent suctioning. Consequently it is likely that infants with repeated or multiple manipulations of the airway may be at higher risk for developing SGCs and should be followed up closely to avoid potential life threatening complications.

\section{PO-0642 NEURODEVELOPMENTAL OUTCOMES OF VLBW CHILDREN AT 6-8 YEARS OF AGE}

P Nuntnarumit, J Intachumpoo. Department of Pediatrics, Faculty of Medicine Ramathibodi Hospital Mahidol University, Bangkok, Thailand

10.1136/archdischild-2014-307384.1283

Background There is growing awareness that the majority of nondisabled survivors encounter more "subtle" problems such as academic under achievement, behavioural problems, and deficits in executive functions.

Objective To compare gross motor function,cognitive function, academic competence and behavioural problems at school age between VLBW children and controls.

Methods We enrolled children aged 6-to 8-year-old, who were born with $\mathrm{BW} \leq 1,500 \mathrm{~g}$ and have been followed-up at our long-term, follow-up clinic. They were tested for cognitive function and academic achievement using Wechsler Intelligence Scale for Children-III(WISC-III) and Wide Range Achievement Test (WRAT). Child Behaviour Checklist for emotional/behavioural assessment was completed by the care givers. Gross motor function was assessed using Gross Motor Function Classification System (GMFCS). 
Results Thirty VLBW children were assessed at mean age of 7.5 years; 30 children born at term (matched for age, sex, and family income) served as controls. WISC-III scores were comparable between the two groups $(99.8 \pm 2.4$ and $105.8 \pm 1.7 ; \mathrm{p}=$ 0.072 in VLBW and control group, respectively) as well as the WRAT scores. GMFCS-mild dysfunction was found only in 2 children (6.7\%) of VLBWgroup. In contrast, VLBW children had more behavioural/emotional problems, especially in attention deficit/hyperactive $(26.7 \%$ in VLBW group vs.3.3\% in controls, $\mathrm{p}=0.026)$.

Conclusions In our cohort study, VLBW children at school age are at higher risk for behavioural/emotional problems, especially in attention deficit compared with children born at term. However, no differences in cognitive, academic, and gross motor function were found.

\section{PO-0643 MACROPHAGE ACTIVATION SYNDROME IN A NEWBORN INFANT BORN TO A MOTHER WITH AUTOIMMUNE DISEASE}

JH Park, SH Kim, DC Jeong, SY Kim. Pediatrics, The Catholic University of Korea College of Medicine, Seoul, Korea

\subsection{6/archdischild-2014-307384.1284}

We reported a newborn infant with macrophage activation syndrome (MAS) born from a mother with positive anti-nuclear (ANA) and anti-SSA/Ro antibodies. The 2,500 g girl was born at $37^{+6}$ weeks of gestation in good condition. Mother had been diagnosed with adult onset Still disease 10 years previously. During pregnancy, she did not take any medication as she was free of symptoms. The baby was admitted due to tachypnea and fever $12 \mathrm{~h}$ after birth. Initial laboratory findings showed mild anaemia with thrombocytopenia, and mild elevation of alanine aminotransferase (ALT). A work-up of infectious aetiology, including agents responsible for congenital infection, was negative. On the 10th hospital day (HD), the baby showed severe abdominal distension caused by hepatosplenomegaly, and persistent, high fever despite empirical antibiotic therapy. We identified positive ANA and anti-SSA/Ro antibodies from the infant, compatible with those found in the mother. The baby's electrocardiography was normal. On the 18th HD, she showed deterioration of overall condition with high ferritin, ALT, and profound

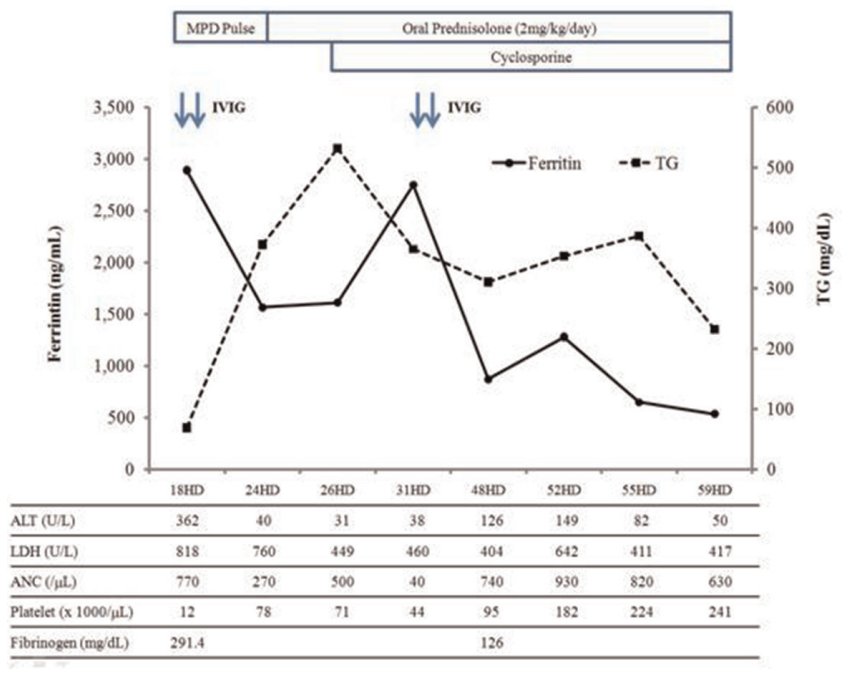

Abstract P0-0643 Figure 1 thrombocytopenia. The baby received intravenous immunoglobulin, steroid (pulse and oral), and cyclosporine. Gene study for perforin, K-ras, and N-ras was negative. Her general condition showed improvement after treatment, although mild fever and organomegaly remained. We maintained high dose steroid and cyclosporine, and all medication was tapered and stopped at 12 weeks of age. We suggest that transplacental transfer of maternal auto-antibodies may be associated with the infant's MAS.

\section{PO-0644 WHITE LIGHT SPECTROSCOPIC TRANSCUTANEOUS MEASUREMENTS OF BILIRUBIN LEVELS IN JAUNDICED INFANTS INCLUDING KRAMER ZONES}

${ }^{1} \mathrm{H}$ Rabe, ${ }^{2} \mathrm{M}$ Olbert, ${ }^{3} \mathrm{~S}$ SobowiecKouman, ${ }^{3} \mathrm{R}$ Fernandez, ${ }^{3} \mathrm{P}$ Amess, ${ }^{4} \mathrm{H}$ Jungmann. ${ }^{1}$ Neonatology, Royal Sussex County Hospital, Brighton, UK; ${ }^{2}$ Research and Development, MBR Optical Systems, Wuppertal, Germany; ${ }^{3}$ Neonatology, Brighton and Sussex University Hospitals, Brighton, UK; ${ }^{4}$ Research and Development, MBR OpticalSystems, Wuppertal, Germany

\subsection{6/archdischild-2014-307384.1285}

Background Jaundice is a common problem affecting $8-10 \%$ of preterm and term newborn babies in the first week of life. Efforts have been made to have non-invasive diagnostic devices available for reliable and quick bedside testing of bilirubin levels in order to avoid painful blood taking and delays due to awaiting results from the laboratory. Several transcutaneous devices can estimate bilirubin levels in the newborn and have mostly been tested in term infants. The correlation co-efficient between transcutaneous and laboratory values have been reported to be 0.46 to 0.89 depending on the device. The device cannot be used after phototherapy has started as bilirubin isomeres are produced. Thus a non-invasive device which can measure after the start of phototherapy in preterm and term infants is warranted.

Objective To establish whether the non-invasive white light spectroscopic (WLS) device (Bilispect (R), MBR Optical Systems, Wuppertal, Germany) can measure bilirubin in the skin of preterm and term jaundiced babies before and after phototherapy.

Methods Prospective single centre study of preterm and term infants who had their bilirubin levels taken for clinical reason in a convenience cohort sample. Best measurement site on skin was determined by comparing WLS measurements at 4 Kramer zones (forehead, sternum, forearm and foot) in 9 infants. Forearm fared best. Prospective WLS measurements were taken within 15 min of the blood test at the forearm of the baby. Statistical comparison was performed by Bland-Altman method. Intra- and inter-observer variability were calculated by using repeated measurements and two observers. The ethics committee issued a favourable opinion and informed consent was obtained from parents.

Results Comparisons were obtained in 47 preterm and term infants (range 24-40 weeks gestation, birth weight 564-4220 g), who had 51 paired bilirubin samples done. Ten were taken after phototherapy had been started. Correlation coefficients for samples taken after phototherapy was 0.87 and 0.9 without phototherapy. Intra-observer and inter-observer variability were 0.67 and 0.69 respectively.

Conclusions The results show a good correlation between the laboratory and non-invasive bilirubin values. The WLS seems to be a suitable method for estimating bilirubin levels after phototherapy has started and particularly in preterm infants. Further field studies re required in order to obtain nomograms for this device. 